\title{
Pinturas y engobes EN la ArauCANía: ANÁlisis COMPOSICIONAL DE VASIJAS DECORADAS EL VERGEL (SUR DE CHILE, IOOO-I 550 D.C.)
}

\author{
Javiera Gajardo A. \\ https://orcid.org/0000-0001-8073-7894 \\ Proyecto FONDECYT 11150397 \\ javiera.mga@gmail.com
}

Resumen

La cerámica pintadalengobada es uno de los elementos más emblemáticos del Complejo El Vergel del Sur de Chile (1000-1550 d.C.). Si bien se ha abordado su variabilidad desde aspectos tipológicos, decorativos y tecnológicos, faltaba integrar más información respecto a las distintas elecciones en su manufactura para observar la distribución de los modos de hacer. Con aquello como objetivo, se procedió a analizar las pinturas y engobes de material cerámico fragmentado pintadolengobado de cinco sitios domésticos de diferentes áreas geográficas. Estas se analizaron mediante LA-ICP-MS, dando cuenta de las distintas "recetas" utilizadas en su confección. Sobre la base de lo anterior, se identificaron distintos grupos composicionales en engobes y pinturas que se comparten en ciertos ejes geográficos y que pueden responder a una circulación de conocimientos, materias primas y/o vasijas entre distintos grupos El Vergel.

Palabras clave: Sur de Chile, Complejo El Vergel, cerámica decorada, pinturaslengobes, LA-ICP-MS.

\section{Abstract}

\section{SLIPS AND PAINTINGS IN ARAUCANÍA: COMPOSITIONAL ANALYSIS OF DECORATED VESSELS FROM EL VERGEL COMPLEX (SOUTHERN CHILE, AD 1000-1550)}

For the Southern Chile El Vergel Cultural Complex (1000-1550 AD), painted/slipped pottery is one of its most emblematic elements. Although its variability has been addressed from typological, decorative and technological aspects, it was necessary to integrate more information regarding the different choices in its manufacture to observe the distribution of the "ways of making" (modos de hacer). With this goal, we proceeded to analyze the paintings and slips of decorated sherds from five domestic sites of different geographical areas. They were analyzed by LA-ICP-MS, identifying the different "recipes" used in their manufacture. Based in this, different compositional groups were identified in slips and paintings, being some of them shared in certain geographic axes. This may be due to a circulation of knowledge, raw materials and/or vessels between different El Vergel groups.

Keywords: Southern Chile, El Vergel Complex, decorated pottery, paints/slips, LA-ICP-MS. 


\section{Introducción}

El Complejo El Vergel se desarrolló en el sur de Chile durante el período Alfarero Tardío (10001550 d.C.) y ocupó diversos espacios, tales como los valles al oriente y poniente de la cordillera de Nahuelbuta, la costa, las islas y parte de la zona cordillerana andina hacia el sur (Aldunate 1989; Reyes 2010; Donoso 2017), desde el río Biobío por el norte, hasta el río Toltén, por el sur. Esta unidad arqueológica es poseedora de una gran variabilidad interna (Dillehay 1990) reflejada en las distintas manifestaciones locales que se han registrado en la zona: construcción de arquitectura pública solo en algunos lugares, sitios arqueológicos más o menos densos, heterogeneidad de patrones funerarios, desarrollo dispar del trabajo en metales y un mayor o menor énfasis en los recursos cultivados (Aldunate 1989, 2005; Campbell 2005, 2011; Roa et al. 2012; Silva 2014; Adán et al. 2016; Campbell y Pfeiffer 2017).

A todos estos elementos se suma el indicador clásico del período Alfarero Tardío: la cerámica de la Tradición Bícroma Rojo sobre Blanco (Adán y Mera 1997; Adán et al. 2005), específicamente del estilo Vergel. Esta posee un estilo visual bastante estandarizado, aunque trabajos recientes han dado cuenta de variaciones decorativas acotadas con una distribución espacial determinada (Adán et al. 2005; Bahamondes 2009), que en conjunto con otras evidencias nos muestran distintas maneras de manufacturar estas vasijas, enmarcadas dentro de un contexto de producción local. De esta manera, era necesario obtener más información respecto a las diferentes elecciones hechas por los alfareros y a las distintas dinámicas que subyacen en la elaboración de estas vasijas. Por esta razón se buscó realizar un estudio que abordara las fuentes de aprovisionamiento y procesamiento de pinturas y engobes, con el fin de obtener un panorama más completo de la distribución de los modos de hacer.

En este sentido, las vasijas decoradas son un producto social resultado de las distintas elecciones tomadas por los alfareros durante la cadena operativa. Estas elecciones están constreñidas por el contexto socio-cultural en el cual son aprendidas y reproducidas (Lemonnier 1992; Stark 1999), generando patrones asociados a una manera "correcta» de hacer las cosas. Estos patrones o «modos de hacer» se expresan en estilos tecnológicos (Lemonnier 1992), los cuales residen en cada etapa del proceso de manufactura y en cada aspecto del objeto manufacturado (Lechtman 1977). A su vez, estos estilos tecnológicos nos permiten identificar límites tecnológicos (Stark 1999), los cuales nos ayudan a inferir agrupaciones sociales más cercanas, reflejándose en un correlato material con modos de hacer compartidos (Falabella y Sanhueza 2005). Incluso, son los aspectos ocultos de las vasijas los que nos acercan a las elecciones que se dan cara a cara entre los alfareros - tales como la selección y procesamiento de materias primas - que hacen referencia a redes de interacción locales y unidades mínimas de organización de la producción alfarera (Gosselain 2000; Falabella et al. 2015).

En este caso de estudio, se tratarán de dilucidar interrogantes en torno a la distribución espacial de ciertos modos de hacer entre grupos pertenecientes al Complejo El Vergel, los cuales se integran en una escala amplia en el sector septentrional de la Araucanía. De esta manera, pinturas/engobes similares pueden sugerir núcleos de enseñanza-aprendizaje cercanos, conocimientos compartidos, o una producción generada en un solo lugar con pigmentos y/o vasijas que circulan por el espacio, expresado en una homogeneidad dentro y entre unidades domésticas. Por otro lado, si encontramos una mayor variabilidad en cuanto a «recetas» de pinturas y engobes, podría inferirse un nivel de producción local-familiar con modos de hacer y conocimientos diferentes entre las comunidades, con una escala de integración más doméstica entre los productores de alfarería; incluso, esta variabilidad entre grupos podría permear dentro de las comunidades mismas.

\section{Estudios previos}

La cerámica fragmentada pintada/engobada analizada se obtuvo en el marco del proyecto FONDECYT 11150397. Previo al análisis de LA-ICP-MS y en relación a otros objetivos de la 


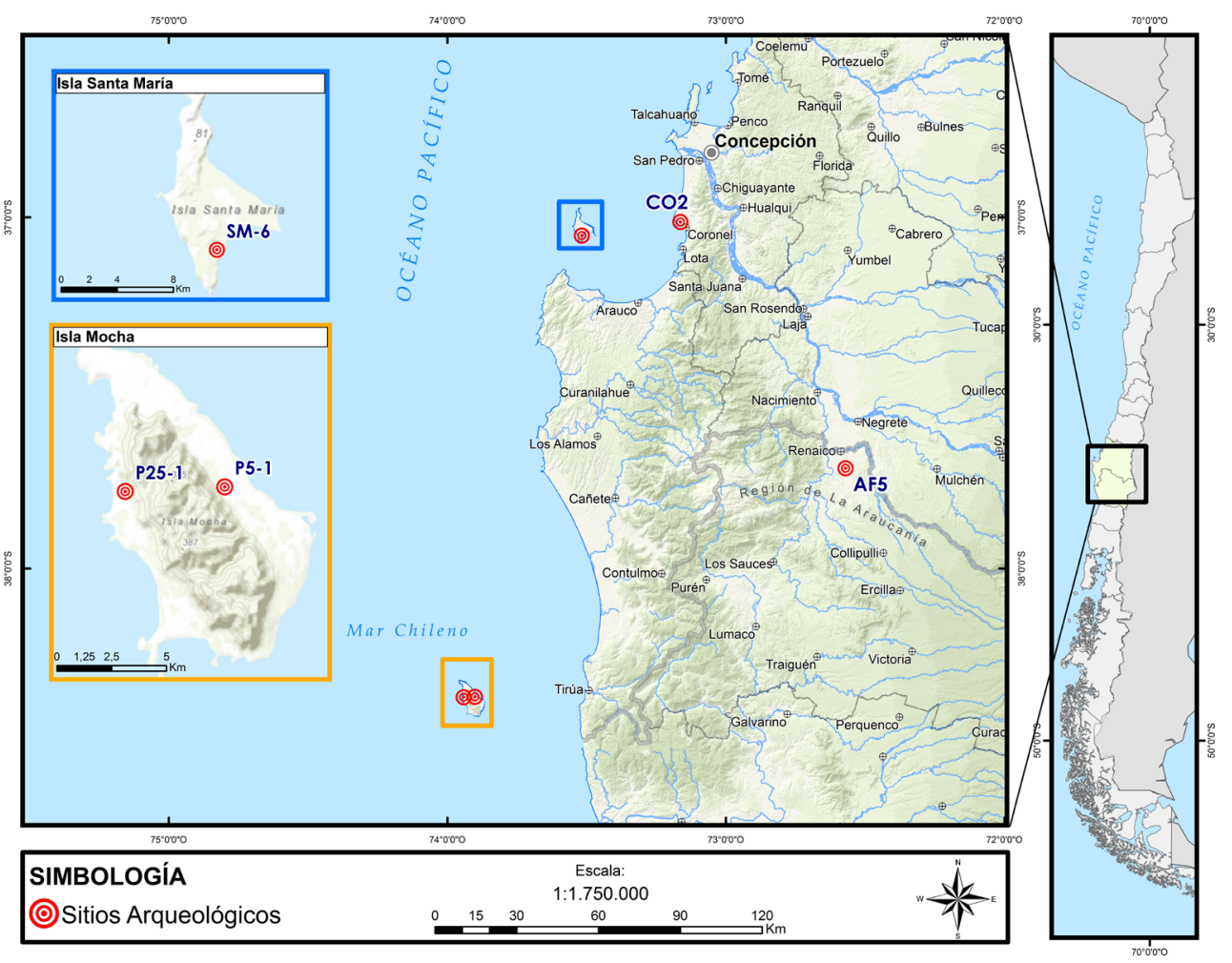

Figura 1. Mapa con localización de los sitios arqueológicos.

investigación, en una primera etapa se realizó un análisis morfológico, de tratamientos de superficie y de pinturas y engobes. Esto con el fin de identificar los segmentos de las vasijas a la que corresponden los fragmentos y eventualmente poder reconstruir categorías morfológicas, inferir tamaños, caracterizar modos de dar acabado a las paredes y determinar la presencia de engobes y pinturas junto a la identificación del color actual de cada una (Gajardo 2017). En una etapa posterior, se buscó caracterizar las pastas utilizadas en la producción de esta cerámica. Para esto, se observó la sección fresca de todos los fragmentos a los que se les pudo hacer un despunte bajo una lupa binocular $(10 \mathrm{x}-40 \mathrm{x})$. Se identificaron los atributos de color y textura de la matriz arcillosa para inferir el uso de arcillas caoliníticas o rojizas, y en cuanto a las inclusiones se observó color, forma, densidad y tamaño. Sobre la base de estos datos, se generaron agrupaciones mayores denominadas familias de pasta (Sanhueza, 1997), los que aluden a las formaciones geológicas de donde eventualmente provienen, en el caso de las arenas (Falabella et al. 1993). En este caso, se pudieron determinar diez familias de pasta en un $76.97 \%(\mathrm{~N}=1795)$ del total de la muestra, las cuales presentaron variantes internas (Gajardo 2017).

\section{3. Área de estudio y sitios}

El área bajo estudio se sitúa en el sector septentrional del sur de Chile, en las provincias de Arauco, Concepción y Malleco y abarca parte de los distintos espacios ocupados por los grupos El Vergel en la región. Específicamente, los sitios trabajados corresponden a contextos domésticos ubicados en distintas zonas: en isla Mocha los sitios P25-1 y P5-1, en isla Santa María el sitio SM-6, al poniente de la Cordillera de Nahuelbuta el sitio Coronel 2 (Co-2) y al oriente de la misma el sitio Aerogenerador F5 (AF5) (Fig. 1). 


\section{Materiales y metodología}

Dentro del total de fragmentos cerámicos pintados/engobados $(\mathrm{N}=2332)$ provenientes de los cinco sitios domésticos mencionados previamente, se seleccionó y analizó una muestra para el análisis de pinturas y engobes $(\mathrm{N}=130)$, la cual presenta engobe rojo $(\mathrm{RE})$, engobe blanco $(\mathrm{BE})$ o pintura roja sobre engobe blanco (R/BE) (Tabla 1). Los fragmentos fueron analizados por Hector Neff (2017) en el Institute for Integrated Research in Materials, Environments and Societies (IIRMES) de California State University-Long Beach mediante ablasión láser acoplada a espectometría de masas, con fuente de plasma de acoplamiento inductivo (LA-ICP-MS), en un ICP-MS cuádruplo Agilent 7500, conectado a un sistema de ablasión láser New Wave UP213. Este espectómetro resulta ser muy efectivo incluso con los engobes y pinturas más delgados (Neff 2012), además de ser muy sensible para un amplio rango de elementos. Fue eficiente en la determinación de grupos composicionales químicos para poder establecer las distintas «recetas» utilizadas en la confección de las vasijas, entendiéndose estas como las distintas elecciones para obtener la materia prima y preparar la mezcla para las pinturas y engobes (Speakman y Neff 2002; Duwe y Neff 2007; Porter y Speakman 2008).

El criterio de selección de los fragmentos se basó, primero, en los distintos grupos de tratamiento pintado/engobado presente en los sitios para poder comparar dentro y entre cada sitio $y$, en segundo lugar, se consideraron las distintas proporciones de familias de pasta realizadas en una etapa previa de la investigación, buscando obtener muestras proporcionales a la frecuencia de pastas en cada sitio.

\begin{tabular}{|l|c|c|c|c|c|}
\hline \multirow{2}{*}{ Sitios } & \multicolumn{5}{|c|}{ GRUPO DECORATIVO } \\
\cline { 2 - 6 } & RE & BE & R/BE & Total & $\begin{array}{c}\text { Total pintados/ } \\
\text { engobados }\end{array}$ \\
\hline AF5 & 18 & 2 & 5 & 25 & 49 \\
\hline CO-2 & 10 & 2 & 18 & 30 & 1080 \\
\hline P25-1 & 20 & 0 & 5 & 25 & 173 \\
\hline P5-1 & 16 & 3 & 6 & 25 & 142 \\
\hline SM-6 & 17 & 0 & 8 & 25 & 888 \\
\hline Total & 81 & 7 & 43 & 130 & 2332 \\
\hline
\end{tabular}

Tabla 1. Muestra analizada mediante LA-ICP-MS por sitio y total de fragmentos pintados/engobados respectivos. $R E=$ rojo engobado; $B E=$ blanco engobado; $R / B E=$ rojo sobre blanco.

El análisis se realizó a nivel elemental directamente sobre las pinturas y engobes en la superficie exterior, interior o exterior e interior de los fragmentos, considerando cada color en cada superficie como un análisis particular. En este trabajo, se obtuvo tres muestras de cada color según fuese el caso. Los fragmentos fueron dispuestos en una atmósfera de helio (He) utilizando un punto de 75 micrones de diámetro con un $80 \%$ de potencia para ser analizados. Así, se pudo determinar la abundancia de 59 elementos químicos minerales (mayores, menores, trazas). Estos datos fueron escaneados en busca de anomalías las cuales fueron removidas. Posteriormente se calcularon promedios con las tres muestras por cada color.

Los datos fueron calibrados mediante los estándares de vidrio NIST SRM614, SRM612 y SRM610, los cuales son apropiados para los elementos traza y otros elementos raros, y con los estándares Little Glass Buttles obsidian y NIST brick clay SRM679 para extender el rango de concentración de varios elementos mayores y menores, con el propósito de obtener valores más precisos de esos elementos (Neff 2017). 


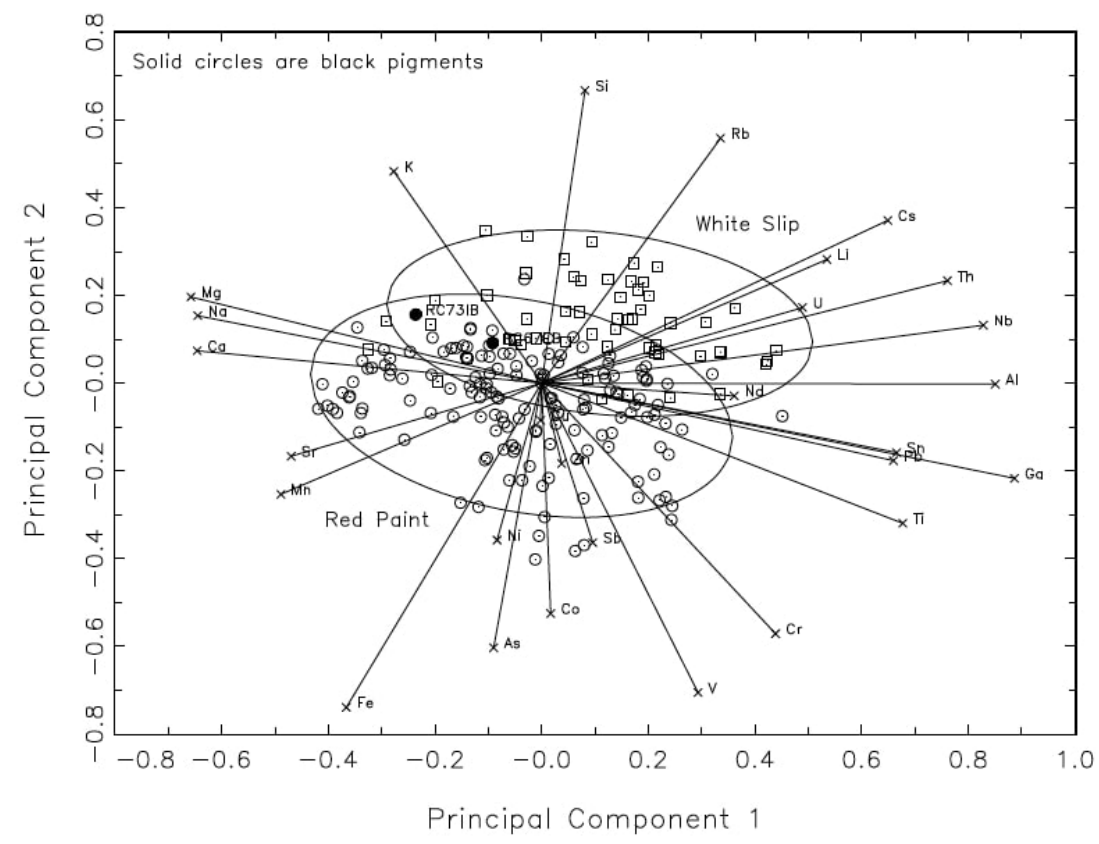

Figura 2. Componentes principales 1 y 2 derivados del PCA de la matriz de correlación de 28 elementos considerados como posibles colorantes, medidos en un total de 205 engobes/pinturas. Los vectores señalan cómo cada elemento contribuye a la configuración de los grupos composicionales. Los elipses representan un nivel de confianza de $90 \%$ en el total de grupos rojos y blancos.

\section{Resultados}

Los resultados del análisis estadístico a partir de LA-ICP-MS entregados por H. Neff (2017) corresponden a análisis de componentes principales (PCA) y análisis canónicos discriminantes. En primer lugar, los análisis de componentes principales, donde los dos primeros explican $47 \%$ de la variabilidad ( $32 \%$ y $15 \%$, respectivamente), nos muestran que aquellos elementos que influyen de manera más clara en la separación de los engobes blancos y pinturas/engobes rojos $(\mathrm{N}=203)$ corresponden al fierro $(\mathrm{Fe})$, vanadio $(\mathrm{V})$ y cromo $(\mathrm{Cr})$ en los rojos, y sílice $(\mathrm{Si})$ y rubidio $(\mathrm{Rb})$ en los blancos (Fig. 2).

Además, los análisis de componentes principales nos indicaron la existencia de ocho grupos composicionales, donde cinco grupos corresponden a las muestras de pintura/engobe rojo y tres grupos a las muestras de engobe blanco, los cuales se separan entre sí en las funciones discriminantes 1 y 2 (Fig. 3). Además, en las funciones discriminantes 2 y 3 (Fig. 4) se aprecia de mejor manera la separación entre los grupos rojos (donde la función discriminante 3 explica $10 \%$ de la variabilidad).

En relación a los grupos rojos específicamente, se observa que la mayor parte de ellos tiene entre un $16-20 \%$ de aluminio (Al), mientras que el grupo designado como Red-1 tiene un promedio aproximado de $8 \%$ de aluminio (Fig. 5). El galio (Ga), elemento que sustituye al aluminio en redes de cristal, también presenta una baja proporción en este grupo respecto a los otros. El aluminio es un elemento mayoritario en las arcillas, por lo que una interpretación a esta diferencia podría deberse a que este grupo representa pigmentos que fueron mezclados con menos arcilla o sin ella en contraste con el resto del conjunto. Esto podría resultar del uso de diferentes fuentes con altos o bajos contenidos arcillosos o bien, podría deberse a prácticas de preparación diferenciadas, mezclando más o menos arcilla con óxidos de fierro. 


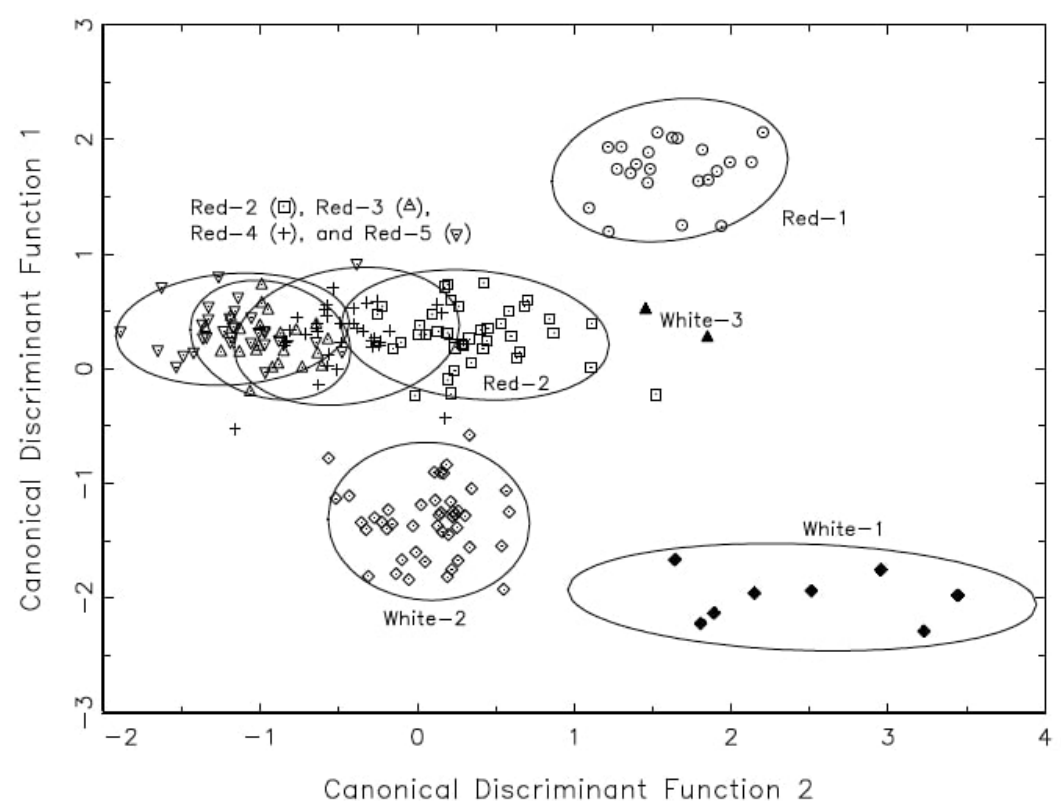

Figura 3. Grupos composicionales $(N=8)$ reconocidos en los datos de pinturalengobe ploteados en las Funciones Discriminantes 1 y 2 derivados de un análisis canónico discriminante sobre los ocho grupos composicionales. Las elipses representan un nivel de confianza de $90 \%$ para la pertenencia en siete de los ocho grupos. Como el grupo White-3 solo tiene dos miembros, no se puede calcular un elipse de confianza.

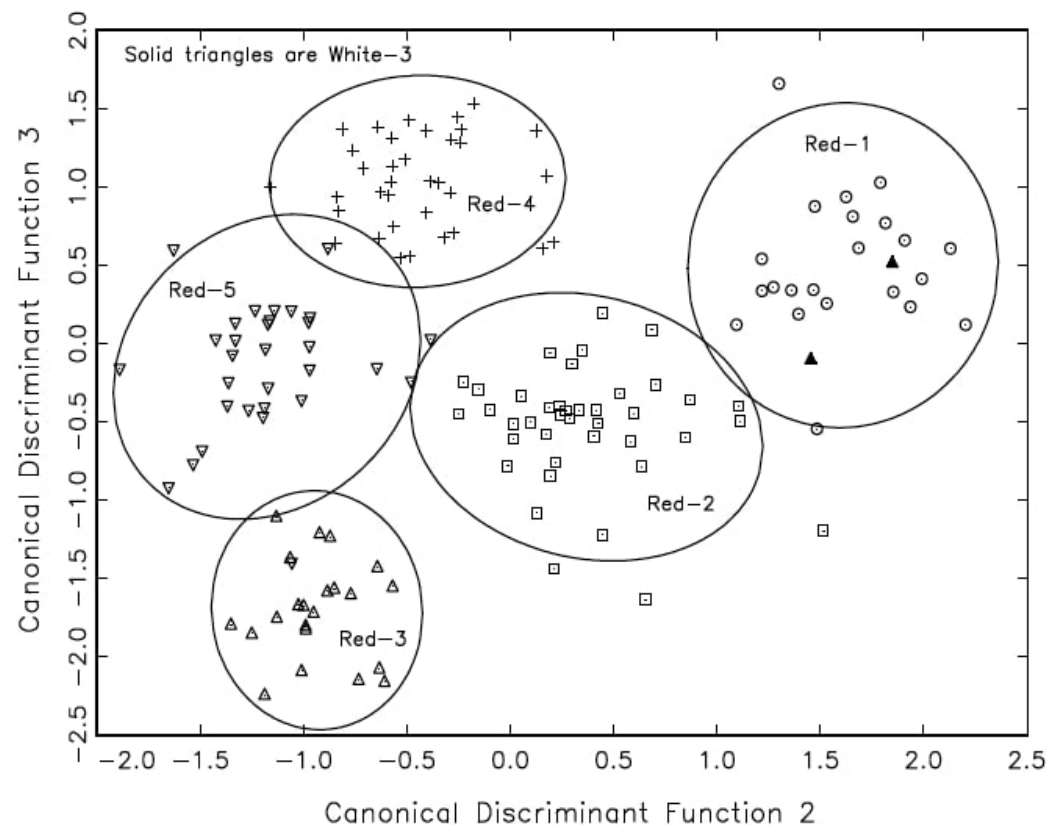

Figura 4. Grupos composicionales de pinturaslengobes rojos ploteados en las Funciones Discriminantes 2 y 3 derivadas de un análisis canónico discriminante del total de ocho grupos. Las elipses representan un nivel de confianza de 90\% para la pertenencia dentro de los grupos. Los triángulos negros sólidos corresponden al grupo White-3. 
Por otro lado, otro de los grupos composicionales denominado Red-3 se diferencia del resto por presentar un muy bajo nivel de estaño $(\mathrm{Sn})$, con valores promedio cercanos a $1 \mathrm{ppm}$, en contraste a los 3-10 ppm en los otros grupos rojos. Esta diferencia podría representar la explotación de una fuente de óxido de fierro con bajas concentraciones de estaño (Fig. 6).

Asimismo, tres de los grupos de pintura/engobes rojos, Red-2, Red-4 y Red-5, tienen composiciones muy similares y se sobreponen en la mayoría de las proyecciones de datos (Figs. 3, 5 y 6). La diferencia entre estos grupos se basa en dos elementos terrestres alcalinos: el calcio (Ca) y el estroncio (Sr) (Fig. 7). El grupo Red-2 destaca por poseer concentraciones muy elevadas de ambos elementos, con concentraciones de calcio en el rango de 3-10\%. Es posible que los altos valores de este elemento se deban a la adición de carbonato de calcio durante la preparación o también podría deberse a diferencias en las vetas naturales disponibles. Por su parte, los grupos Red-4 y Red-5 poseen distintas líneas de correlación. La disimilitud entre ambos se puede interpretar como una diferencia en la fuente de obtención de materia prima, donde las fuentes del grupo Red-5 están más enriquecidas en calcio y estaño en comparación a Red-4.

En lo que respecta a los engobes blancos, los tres grupos corresponden a White-1, White-2 y White-3. Este último está compuesto por solo dos especímenes (Figs. 8 y 9) y ambos presentan concentraciones de fierro más consistentes con las pinturas/engobes del grupo Red-2, lo cual podría deberse a una contaminación por ablación parcial de la matriz arcillosa subyacente.

Por su parte, el grupo White-1 presenta concentraciones más altas de calcio y estroncio en relación a White-2, con un promedio de $16 \%$ y $8 \%$, respectivamente (Fig. 8). El calcio está altamente correlacionado con el estroncio, el cual sustituye al calcio en los carbonatos (Fig. 7). Estas concentraciones resultan ser las más altas entre todas las pinturas y engobes, lo que nos podría indicar que se agregó carbonato de calcio como colorante en los engobes para formar esta «receta». En contraparte, el grupo White-2 es bajo en calcio, pero tiende a presentar concentraciones más altas de aluminio respecto a White-1, con un promedio de $20 \%$ y $10 \%$, respectivamente (Fig. 9). Esto nos podría sugerir que el engobe con la «receta» White-2 podría corresponder a una arcilla caolinítica, la cual genera tonos blanquecinos con un proceso de cocción con suficiente oxidación. No obstante, como ciertos engobes del grupo White-1 presentan hasta un $16 \%$ de aluminio en los tres sitios insulares, se puede suponer que se emplearon estrategias alternativas e incluso combinadas para lograr el color deseado.

Al observar la distribución de los grupos composicionales en los distintos sitios, se puede establecer que hay una distribución no aleatoria en el espacio, donde algunos grupos se comparten entre sitios y otros son exclusivos de algunos. A grandes rasgos, se aprecian diferencias entre los sitios insulares y aquellos del continente (Tabla 2). En cuanto a los sitios continentales, tanto AF5 como Co-2 poseen engobes blancos compuestos en un 100\% del grupo composicional químico White-2. También comparten el grupo Red-5 en las pinturas/engobes rojos, aunque con porcentajes muy disímiles (100\% y 10.71\%, respectivamente). En Co-2 se registraron también los grupos Red-2 y Red-4 con un $10.71 \%$ y $78.57 \%$, respectivamente.

En cuanto a los sitios insulares, el grupo White-1 está presente en todos (P25-1, P5-1 y SM-6), aunque también presentan el grupo White-2 que caracteriza a los sitios continentales. Además, el sitio P5-1 es el único que registra el grupo White-3. Con respecto a las pinturas/engobes rojos, el grupo Red-1 se encuentra exclusivamente en los sitios de isla Mocha P25-1 y P5-1, y solo este último presenta el grupo Red-4 entre los sitios insulares. El sitio SM-6, en isla Santa María, es el único que registra el grupo Red-3 siendo el más dominante (64.00\%). Además, los tres sitios insulares comparten pinturas/engobes del grupo Red-2, y los sitios P5-1 y SM-6 contienen también, aunque en menor proporción, el grupo Red-5 en sus pinturas/engobes. 


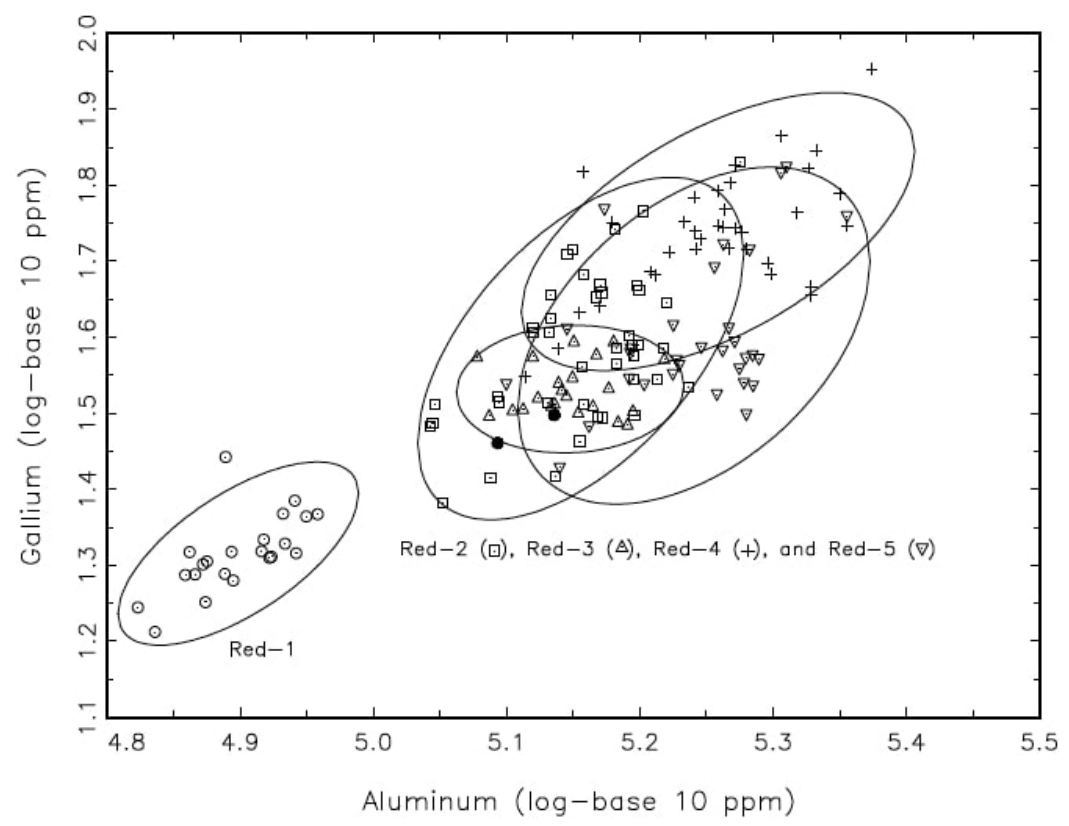

Figura 5. Gráfico bivariado de concentraciones logaritmicas de aluminio y galio en los cinco grupos de pinturaslengobes rojos. Las elipses representan un nivel de confianza del $90 \%$ de pertenencia en los cinco grupos.

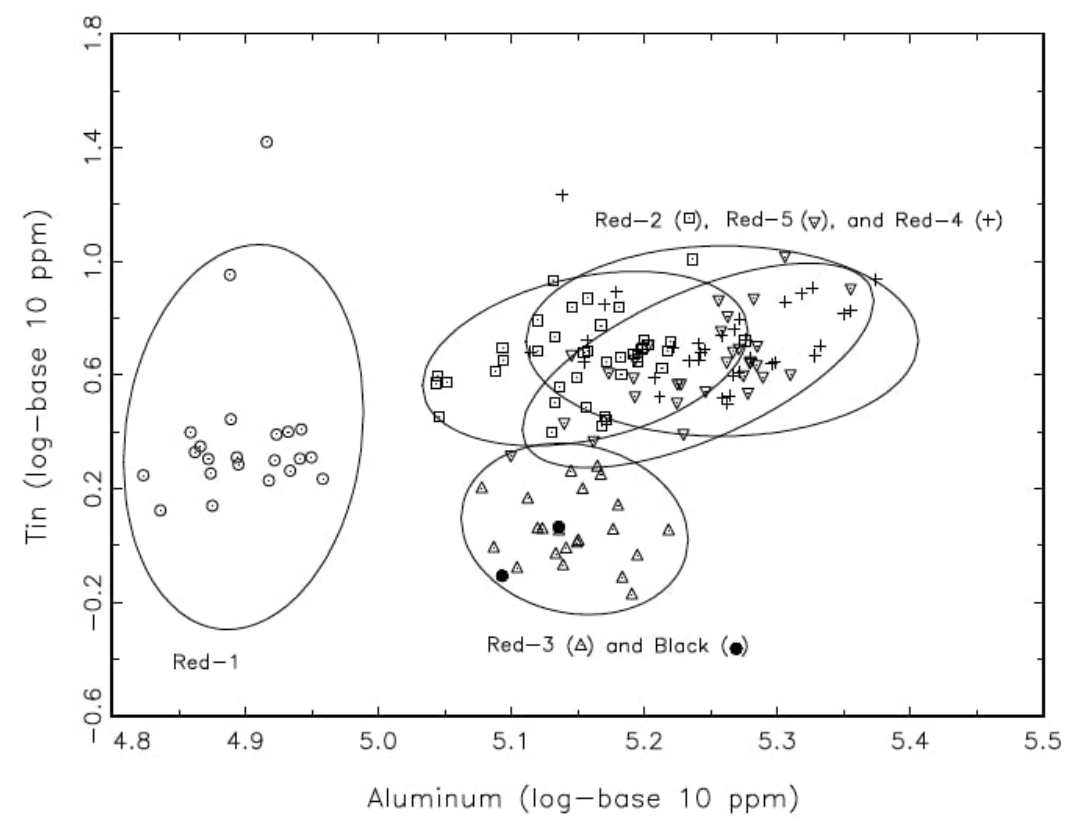

Figura 6. Concentraciones logaritmicas de aluminio y estaño en los grupos de pinturalengobe rojos. Las elipses representan un nivel de confianza del 90\% para la adhesión de los grupos. 


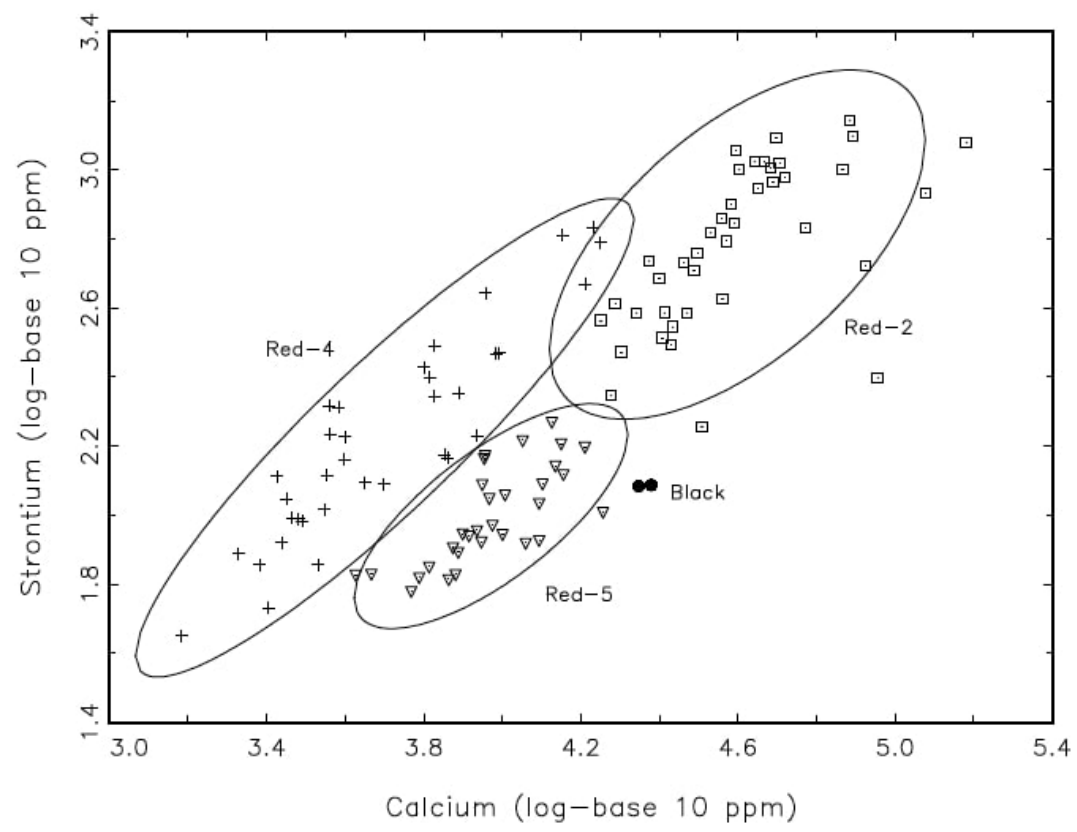

Figura 7. Concentraciones logaritmicas de calcio y estroncio en los grupos Red-2, Red-4 y Red-5. Las elipses representan un nivel de confianza del $90 \%$ para la adhesión de los grupos.

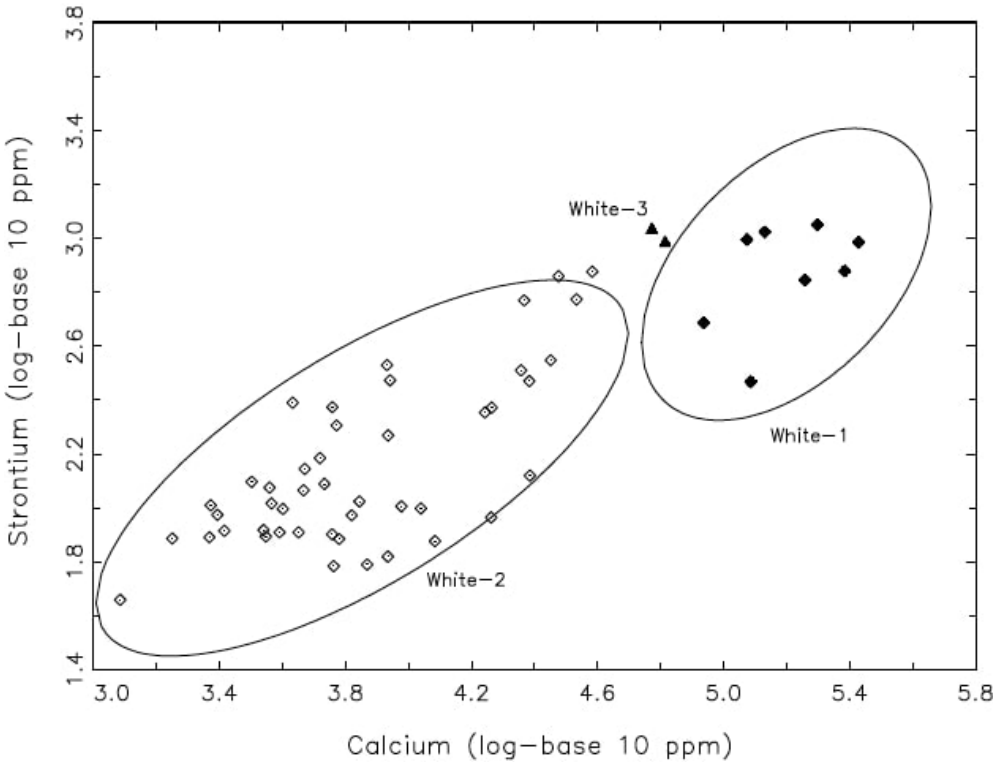

Figura 8. Concentraciones logaritmicas de calcio y estroncio en los grupos de engobes blancos. Las elipses representan un nivel de confianza de $90 \%$ para la adhesión de los grupos. 


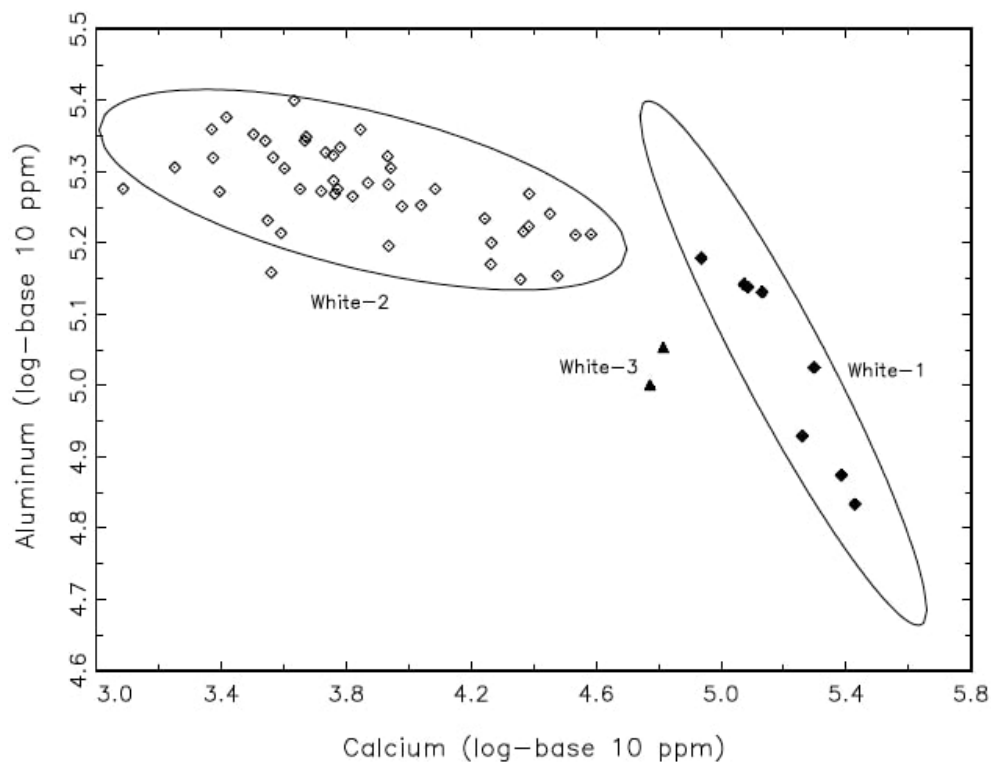

Figura 9. Concentraciones logaritmicas de calcio y aluminio en los grupos de engobes blancos. Las elipses representan un nivel de confianza del $90 \%$ para la adhesión de los grupos.

\begin{tabular}{|c|c|c|c|c|c|c|c|c|c|c|c|c|}
\hline \multirow{4}{*}{$\begin{array}{l}\text { Grupos com- } \\
\text { posicionales }\end{array}$} & \multicolumn{12}{|c|}{ Sitios } \\
\hline & \multicolumn{4}{|c|}{ Continentales } & \multicolumn{6}{|c|}{ Insulares } & \multirow{2}{*}{\multicolumn{2}{|c|}{ Total }} \\
\hline & \multicolumn{2}{|c|}{ AF5 } & \multicolumn{2}{|c|}{$\mathrm{CO}-2$} & \multicolumn{2}{|c|}{ P25-1 } & \multicolumn{2}{|c|}{ P5-1 } & \multicolumn{2}{|c|}{ SM-6 } & & \\
\hline & $\mathrm{N}$ & $\%$ & $\mathrm{~N}$ & $\%$ & $\mathbf{N}$ & $\%$ & $\mathbf{N}$ & $\%$ & $\mathbf{N}$ & $\%$ & $\mathbf{N}$ & $\%$ \\
\hline Red-1 & 0 & 0.00 & 0 & 0.00 & 10 & 41.67 & 4 & 22.22 & 0 & 0.00 & 14 & 11.97 \\
\hline Red-2 & 0 & 0.00 & 3 & 10.71 & 14 & 58.33 & 9 & 50.00 & 7 & 28.00 & 33 & 27.35 \\
\hline Red-3 & 0 & 0.00 & 0 & 0.00 & 0 & 0.00 & 0 & 0.00 & 16 & 64.00 & 16 & 13.68 \\
\hline Red-4 & 0 & 0.00 & 22 & 78.57 & 0 & 0.00 & 4 & 22.22 & 0 & 0.00 & 26 & 22.22 \\
\hline Red-5 & 23 & 100 & 3 & 10.71 & 0 & 0.00 & 1 & 5.56 & 2 & 8.00 & 29 & 24.79 \\
\hline Total rojo & 23 & 100 & 28 & 100 & 24 & 100 & 18 & 100 & 25 & 100 & 118 & 100 \\
\hline White-1 & 0 & 0.00 & 0 & 0.00 & 3 & 60.00 & 2 & 22.22 & 2 & 28.57 & 7 & 14.58 \\
\hline White-2 & 7 & 100 & 20 & 100 & 2 & 40.00 & 5 & 55.56 & 5 & 71.43 & 39 & 81.25 \\
\hline White-3 & 0 & 0.00 & 0 & 0.00 & 0 & 0.00 & 2 & 22.22 & 0 & 0.00 & 2 & 4.17 \\
\hline Total blanco & 7 & 100 & 20 & 100 & 5 & 100 & 9 & 100 & 7 & 100 & 48 & 100 \\
\hline
\end{tabular}

Tabla 2. Porcentajes de grupos composicionales por sitio. Se indica en negrita el grupo composicional blanco y rojo más representado en cada sitio.

\section{Discusión: producción local, circulación de conocimientos y/o materias primas}

Las evidencias presentadas permiten ampliar el conocimiento sobre el contexto de producción de las vasijas pintadas/engobadas y, sobre todo, a comprender aún más las distintas dinámicas sociales que subyacen a estos contextos de producción. De esta manera, las vasijas pintadas/engobadas demuestran ser un elemento muy dinámico dentro de los grupos El Vergel. Por un lado, poseen un 
acabado común y un estilo visual bastante estandarizado, donde son justamente los aspectos decorativos los que generan una unidad dentro de este complejo identificándolos a un nivel regional (Bahamondes 2009). No obstante, bajo esta aparente unidad y homogeneidad, estudios previos han dado cuenta de que la manufactura de estas vasijas posee diversos modos de hacer. Entre varios aspectos, destaca la identificación de distintos tipos decorativos asociados a ciertas localidades (Adán et al. 2005; Bahamondes 2009); también se ha observado una variabilidad en las pastas utilizadas que refleja parte de las distintas elecciones hechas por los alfareros en relación a la selección y procesamiento de estas (Bahamondes 2009; López, 2016; Donoso 2017; Gajardo 2017), también hay diversidad en los tipos de trazos ejecutados (Reyes 2010), en las terminaciones morfológicas y en la calidad de los engobes y pinturas (Bahamondes 2009). De igual modo resalta la alta variabilidad de colores en las pinturas y engobes (Reyes 2010; Gajardo 2017) que han demostrado estar sujetos a las condiciones y temperaturas de cocción, lo cual fue constatado en un experimento posterior al análisis mediante LA-ICP-MS (Gajardo 2017). En este caso, se sometió una muestra de ocho fragmentos con engobe rojo provenientes de los sitios P25-1 y P5-1 a un proceso de recocción en un horno eléctrico en un ambiente oxidante a $880^{\circ} \mathrm{C}$. Previo a la recocción, los fragmentos presentaban seis colores diferentes (2.5YR 2.5/4, 2.5YR 3/4, 2.5YR 4/6, 2.5YR 4/8, 2.5YR 5/6, $10 \mathrm{R} 3 / 6)$ y al finalizar el proceso se obtuvieron dos colores, donde cuatro fragmentos presentaron un rojo claro (2.5YR 5/8) y cuatro fragmentos presentaron un rojo oscuro (2.5YR 3/4).

En definitiva, lo anterior nos estaría indicando que no hay una tendencia significativa que refleje una manera "correcta» de hacer las vasijas ampliamente compartida, sino más bien se evidencian distintas elecciones tecnológicas que denotarían parte de la libertad de los artesanos al momento de confeccionarlas, aunque siempre dentro de una pauta de configuración básica que busca un aspecto común (Bahamondes 2009).

En este sentido, la variabilidad observada en este estudio junto con otras investigaciones previas (Reyes 2010; López 2017) han llevado a inferir que no habría un centro productivo único, sino por el contrario, se sugiere un nivel de producción local en cada comunidad. Incluso en los sitios domésticos de isla Mocha se han identificado pulidores, arcilla sin cochurar y probables hornos (Adán y Mera 1997; Campbell 2011; López 2016; Sánchez 1997), evidencia que apoya este planteamiento. Los grupos Vergel que producen y utilizan esta cerámica se ordenan en pequeñas unidades políticas de tipo transigualitario, en donde las unidades domésticas no son iguales entre sí, sino que poseen algún grado de diferenciación social, pero sin generar diferencias jerárquicas a la vez de tener la capacidad de organizarse supralocalmente (Campbell 2011). Esto permite explicar la variabilidad observada en la cerámica pintada/engobada y sus distintos modos de hacer, aunque con elementos decorativos y morfológicos similares que permiten integrarlos bajo una misma tradición alfarera, que busca un aspecto común (Bahamondes 2009).

Es en este escenario que cobra relevancia el estudio realizado. La identificación de los grupos composicionales o «recetas» utilizadas para el tratamiento de superficie (ya sea pintura o engobe), junto a la distribución de estas, nos muestra un panorama muy dinámico, con relaciones sociales que van más allá de la unidad doméstica y que nos enseñan el juego de relaciones sociales insertas en estas vasijas. Además, estas «recetas», al ser parte de los aspectos que no podemos ver a simple vista en las vasijas, remiten a interacciones más cercanas entre los miembros de los grupos alfareros y poseen distintos alcances espaciales. De esta manera, podemos observar algunos conocimientos y/o materias primas que no circulan en el espacio y se quedan dentro de un contexto acotado como en el caso de las islas, mientras que también se distinguen interacciones más amplias que tienen configuraciones espaciales en más de un sentido, dando cuenta de distintos niveles de interacción entre los grupos.

Para la interpretación de estos datos nos basamos en dos postulados que se utilizan frecuentemente en los estudios de procedencias de materias primas. El primero corresponde al «criterio de abundancia» (Bishop et al. 1982), el cual nos indica que un grupo composicional que está representado en gran medida en un sitio arqueológico sugiere que pertenece al perfil químico de 
la fuente característica explotada en esa localidad (Falabella y Andonie 2011). El segundo corresponde al "postulado de procedencia» (Weigand et al. 1977), donde las diferencias entre los grupos composicionales remiten a distintas fuentes. Esto se sustenta en que las diferencias dentro de una misma fuente son menores a las diferencias entre distintas fuentes.

En primer lugar, se observa que en ambas islas hay elecciones diferenciadas en la preparación de sus pinturas y engobes, y que están circunscritas a las islas mismas. En los sitios P25-1 y P5-1 se infiere que hay una circulación de conocimientos y/o materias primas entre ambos, pues los dos utilizan la «receta» Red-1 en sus pinturas/engobes rojos, el cual no está presente en los otros sitios. Sus bajos porcentajes de aluminio $(8 \%)$ permiten inferir que habría prácticas de preparación diferenciadas donde se habría realizado una mezcla con menos arcilla o se habría utilizado una fuente con bajos niveles de este elemento. Por otro lado, en el sitio SM-6 predomina el grupo Red-3, donde los alfareros explotaron una veta particular, la cual probablemente se encuentra dentro de la isla por encontrarse representada solo en este sitio en altos porcentajes y por su característico nivel bajo en estaño.

En un segundo nivel más amplio, se distinguen elecciones similares que agrupan a los sitios insulares entre sí. Los tres sitios comparten la preparación de engobes blancos utilizando la «receta» White-1, la cual posee niveles altos de carbonato de calcio. Sobre la base de la composición geológica de ambas islas, se pudo establecer que las dos presentan yacimientos de coquinas (Gajardo y Alfaro 1980; López et al. 2003), por lo que hay fuentes disponibles para explotar en ambos contextos y podría tratarse de un conocimiento que circula por las islas. Además, en los tres sitios se identificaron fragmentos con el grupo composicional White-1 con altos niveles de aluminio, lo que sugiere que en ciertos casos se agregaron arcillas caoliníticas en su preparación. Estas similitudes resultan muy interesantes, ya que hay una distancia no menor que separa ambas islas, y la península de Arauco es un rasgo que interrumpe la conexión directa entre ambas. La integración de más sitios costeros resultaría necesaria para determinar si hay una transmisión vía terrestre y si es un conocimiento compartido con grupos del continente, o bien un conocimiento restringido para las islas determinado por el acceso a las fuentes de carbonato de calcio.

Una tercera configuración que se aprecia permite agrupar a los sitios cercanos a la costa, los cuales presentan una mayor diversidad. En las pinturas/engobes rojos, los sitios Co-2, P25-1, P5-1 y SM-6 comparten el grupo Red-2, el cual sugiere una probable circulación de esta «receta» o de su materia prima desde la zona insular hacia el continente. Esto se infiere, por un lado, porque este grupo químico se caracteriza por poseer altos niveles de estroncio y calcio, lo que permite inferir una adición de carbonato de calcio, algo ya evidenciado en la preparación de la «receta» White-1. Por otra parte, este grupo químico está representado en altos porcentajes en P25-1 y P5-1 y en menor medida en SM-6, estando solo en un $10.71 \%$ en Co-2.

Otra configuración que merece ser destacada se da entre Co-2 y P5-1, quienes comparten la «receta» de pintura/engobe rojo Red-4. Este grupo está representado en un $78.57 \%$ y en un $22.22 \%$, respectivamente. Esto permite sugerir una procedencia desde el continente hacia el sitio de isla Mocha. Esto resulta muy interesante, pues la distancia entre ambos sitios es considerable. Sin embargo, datos etnohistóricos dan cuenta también de la asociación que habría entre los caciques de esta isla y el intercambio con el continente (v.g. Rosales 1674[1877]), donde se llega a pensar que la incorporación de elementos foráneos a la isla reforzaría y mostraría «las conexiones extra-locales del cacique» (Campbell 2011: 261). Incluso, se han establecido diferencias en los sectores norte y sur de la isla, donde el sitio P5-1 presentaría una diferenciación social sutil respecto de los otros sitios del norte, tratándose de un «contexto más privilegiado o con un status más alto» (López 2016: 68). De hecho, el sitio P5-1 es el que presenta la mayor diversidad de «recetas», integrando algunas provenientes de $\mathrm{Co}-2$ y de AF5, indicando relaciones e interacciones con grupos de distintos espacios, incluso muy alejados espacialmente. 
Por otra parte, en otro plano más amplio se observa una cuarta configuración entre los sitios, que agrupa a la vertiente oriental y occidental de la cordillera de Nahuelbuta y hasta la costa. Esto se evidencia en el uso compartido de la "receta» Red-5 en las pinturas/engobes rojos. Las vasijas que presentan este grupo químico predominan en un 100\% en el sitio AF5, mientras que los porcentajes van disminuyendo a medida que aumenta la distancia desde el valle hacia la costa, lo que nos permite suponer su procedencia en el interior. En este sentido, destacan las barreras geográficas que pueden estar mediando e influyendo en las interacciones entre los grupos de ambas vertientes, como lo sería la cordillera de Nahuelbuta y la franja de mar que separa al espacio insular. De este modo, se ve que este grupo está representado en un $10.71 \%$ en Co-2, en un $8.00 \%$ en SM-6 y en un 5.56\% en P5-1, estando ausente en P25-1.

Finalmente, se observa un nivel de integración aún más amplio que integra a todos los sitios. Esto se ve reflejado en la preparación compartida de engobes blancos utilizando la «receta» White-2. Si bien en los sitios continentales es la única «receta» presente, en las islas se confecciona en paralelo o en conjunto con el grupo White-1. El alto porcentaje de aluminio nos sugiere que se trataría de arcillas caoliníticas, y las fuentes de caolín abundan en la región (Gajardo y Alfaro 1980; López et al. 2003), por lo que hay un modo de hacer ampliamente distribuido y compartido entre los sitios, con fuentes potencialmente disponibles para todos los productores de alfarería.

En síntesis, ciertos aspectos de estas vasijas presentan una alta variabilidad que nos sugieren una menor integración entre alfareras(os). No obstante, otros elementos indican interacciones dadas en un nivel supradoméstico, tal como ocurre con los aspectos decorativos que nos permiten agrupar las vasijas decoradas bajo una misma tradición alfarera (Adán y Mera 1997), aludiendo a fronteras sociales más amplias que no necesariamente implican interacciones regulares entre sus partes (Stark 1999). Es en esta dinámica, entre relaciones locales y supralocales, que los resultados expuestos a partir del análisis de LA-ICP-MS nos ayudan a comprender mejor el panorama social. Si bien estas «recetas», al ser parte de los aspectos ocultos, debieran reflejar redes de interacción locales (Gosselain 2000), también nos muestran otros alcances más amplios: por un lado, son reflejo de interacciones que se dan en espacios más circunscritos como en el caso de las islas, pero también nos revelan redes más amplias que se dan en ciertos ejes espaciales y refuerzan el planteamiento de la existencia de relaciones sociales reiteradas a un nivel supradoméstico. Estas interacciones pueden deberse a una circulación de conocimientos, materias primas y/o núcleos de enseñanza-aprendizaje cercanos.

\section{Conclusiones y proyecciones}

El objetivo de este trabajo era evaluar la variabilidad de la cerámica pintada/engobada El Vergel, y el análisis realizado nos permitió discutir tanto el contexto de producción como las relaciones sociales que subyacen en los procesos de manufactura y uso de estas vasijas. El análisis de LA-ICP-MS nos mostró que hay distintos modos de hacer entre los grupos, donde algunos se comparten y otros no. En este aspecto, solo las islas presentan algunos conocimientos y/o materias primas que quedan circunscritas al espacio insular, aunque es necesario integrar y analizar más sitios de las islas y costa para ver si efectivamente esto se mantiene de este modo, teniendo en consideración que todos los sitios están interactuando con otros grupos, e incluso, los sitios de la costa son los que poseen una mayor diversidad de «recetas». En este aspecto, el sitio P5-1 es el más diverso, mientras que al oriente de la cordillera de Nahuelbuta se observa un panorama más homogéneo que posee relaciones menos frecuentes con los sitios de la otra vertiente. Sin embargo, es necesario considerar que la muestra de fragmentos decorados del sitio AF5 es muy baja respecto a los otros, lo que dificulta su comparación con los otros sitios y también ofrece un panorama muy sesgado de las dinámicas del interior, por lo que se hace necesario integrar más sitios de la vertiente oriente. 
Por otra parte, los análisis arqueométricos siempre se ven enriquecidos al complementarlos entre sí, y la discusión se puede profundizar aún más si así se realiza. En este sentido, el análisis de LA-ICP-MS nos permite suponer al menos una circulación de conocimientos y/o materias primas, pero no podemos establecer la circulación de vasijas pintadas/engobadas completas. Sería necesario incluir análisis petrográficos para determinar de mejor manera si las pastas utilizadas son locales o foráneas, y así poder establecer de manera más precisa la circulación de las materias primas y eventualmente vasijas. Además, esto debe ir a la par de una profundización en estudios geológicos, con el fin de tener un panorama más completo respecto a las posibles fuentes de pigmentos, arcillas y antiplásticos y así tener más información respecto a la cadena operativa de estas vasijas.

Los resultados con este análisis son una primera aproximación que debe ser más desarrollada a futuro, y es necesario integrar más sitios y complementarlo con más análisis para seguir profundizando y reconstruyendo de manera más certera las dinámicas sociales que hay detrás de estas vasijas y detrás de los grupos humanos que las manufacturaron y utilizaron. Por otro lado, también es necesario tener en consideración la variable cronológica en esta variabilidad observada, ya que esta claramente debe responder al factor temporal. No obstante, los fechados por termoluminiscencia (TL) que se poseen en cada sitio no permiten segregar lo suficiente, pues estos poseen rangos de errores amplios y además no se han realizado con la función de fechar fragmentos con distintas «recetas», por ejemplo. Por ende, es necesario considerar en el futuro trabajos que aborden esta perspectiva, para comprender mejor esta transmisión de conocimientos, materias primas y/o vasijas en una escala temporal. En definitiva, este análisis abre muchas posibilidades que requieren ser abordadas con estudios más sistemáticos para seguir contribuyendo al estudio de los conjuntos cerámicos y las personas que las manufacturaron y utilizaron durante el período Alfarero Tardío en el sector septentrional de la Araucanía.

\section{Agradecimientos}

Este estudio se realizó dentro del proyecto FONDECYT 11150397, agradeciendo especialmente a Roberto Campbell por la oportunidad y apoyo entregado. Nuestros agradecimientos también a Fernanda Falabella por guiar este estudio, como también a Mauricio Uribe y Lorena Sanhueza, cuyos comentarios ayudaron a dar forma a este trabajo. Los análisis de LA-ICP-MS fueron realizados en el Institute for Integrated Research in Materials, Environments and Societies (IIRMES) de California State University-Long Beach por Hector Neff. Los fragmentos analizados fueron facilitados por el Museo de Historia Natural de Concepción (Marco Sánchez y Mauricio Massone) (sitio SM-6), Francisco Bahamondes (sitio Co-2), Francisco Vergara (sitio AF5), y proyectos NSF BCS-0956229 y FONDECYT 3130515 (Roberto Campbell) (sitios P5-1 y P25-1).

\section{REFERENCIAS}

Adán, L. y R. Mera (1997). La tradición cerámica bicroma rojo sobre blanco en la región centro-sur: Los estilos vergel y valdivia. Una propuesta tipológica morfológica-decorativa de la alfarería, Informe Final Proyecto Fondecyt 1950823.

Adán, L., Mera, R., Uribe, M. y M. Alvarado (2005). La tradición bícroma rojo sobre blanco en la región sur de Chile: los estilos decorativos Valdivia y Vergel, Actas del XVI Congreso Nacional de Arqueología Chilena, 339-410, Valdivia.

Adán, L., Mera, R., Navarro, X., Quiroz, D. y M. Sánchez (2016). Historia prehispánica en la región centro-sur de Chile: cazadores-recolectores holocénicos y comunidades alfareras (ca. 10.000 años a.C. a 1.550 años d.C.), en: F. Falabella, M. Uribe, L. Sanhueza, C. Aldunate y J. Hidalgo (eds.), Prehistoria en Chile: Desde sus primeros habitantes hasta los incas, 401-442, Editorial Universitaria, Santiago.

Aldunate, C. (1989). Estadio alfarero en el sur de Chile, en: V. Schiappacasse, H. Niemeyer, C. Aldunate e I. Solimano (eds.), Culturas de Chile: prehistoria. Desde sus origenes hasta los albores de la conquista, 329-348, Editorial Andrés Bello, Santiago. 
Aldunate, C. (2005). Una reevaluación del Complejo Cultural El Vergel. Actas del XVI Congreso Nacional de Arqueología Chilena, 331-336, Valdivia.

Bahamondes, F. (2009). La cerámica prehispánica tardía de Araucanía septentrional: el complejo arqueológico El Vergel y su relación con la hipótesis del proceso de andinización, Memoria para optar al título de arqueólogo, Universidad de Chile, Santiago.

Bishop, R., Rands, R. y G. Holley (1982). Ceramic compositional analysis in archaeological perspective, en: M.B. Schiffer (ed.), Advances in Archaeological Method and Theory, 275-330, Academic Press, New York.

Campbell, R. (2005). El trabajo de metales en El Vergel: Una aproximación desde Isla Mocha, Actas del XVI Congreso Nacional de Arqueología, 379-387, Valdivia.

Campbell, R. (2011). Socioeconomic differentiation, leadership and residential patterning at an Araucanian chiefly center (Isla Mocha, AD 1000-1700), tesis de doctorado, University of Pittsburgh, Pittsburgh.

Campbell, R. y M. Pfeiffer (2017). Early public architecture in Southern Chile: Archaeological and pedological results from the Mocha Island mounds and platform complex, Latin American Antiquity 284(4), 1-20.

Dillehay, T. (1990). Araucania: Presente y Pasado, Editorial Andrés Bello, Santiago.

Donoso, S. (2017). La cerámica doméstica Pitrén y El Vergel: continuidades y cambios de una práctica tradicional en el entorno del lago Villarica (Región de la Araucanía), Memoria para optar al título de Arqueóloga, Universidad de Chile, Santiago.

Duwe, S. y H. Neff (2007). Glaze and slip pigment analyses of Pueblo IV period ceramics from east-central Arizona using time of flight-laser ablation-inductively coupled plasma-mass spectrometry (TOF-LA-ICP-MS), Journal of Archaeological Science 34, 403-414. https://doi.org/10.1016/j.jas.2006.06.001

Falabella, F., Deza, A., Román, A. y E. Almendras (1993). Proporciones morfológicas y comportamiento mecánico de la alfarería prehispana de Chile Central: un nuevo enfoque metodológico, Informe Fondecyt 91-1029.

Falabella, F. y L. Sanhueza (2005). Interpretaciones sobre la organización social de los grupos alfareros tempranos de Chile central: Alcances y perspectivas, Revista de Antropología 18, 105-133.

Falabella, F. y O. Andonie (2011). Análisis por activación neutrónica instrumental de alfarería Aconcagua en el valle del río Maipo, Chile, en L. Vetter, R. Vega-Centeno, P. Olivera, y S. Petrick (eds.), II Congreso Latinoamericano de Arqueometría, 215-228, Lima.

Falabella, F., Sanhueza, L., Correa, I., Fonseca, E., Roush, C. y M. Glascock (2015). Tradiciones tecnológicas del Período Alfarero Temprano de Chile Central: Un estudio de bordes, materias primas y pastas de vasijas de cocina en la microrregión de Angostura, Revista de Antropología Chilena Chungara 46(4), 39-102.

Gajardo, J. (2017). Variabilidad de la cerámica pintadalengobada El Vergel en el sector septentrional de la Araucanía, Memoria para optar al título de arqueóloga, Universidad de Chile, Santiago.

Gajardo, A. y G. Alfaro (1980). Mapa metalogénico de la Región del Bío-Bío, Instituto de Investigaciones Geológicas, Santiago.

Gosselain, O. (2000). Materializing identities: an African perspective, Journal of Archaeological Method and Theory 7(3), 187-217.

Lechtman, H. (1977). Style in technology: some early thoughts, en H. Lechtman y R. Merrill (eds.), Material culture: styles, organization, and dynamics of technology, 3-20, American Ethnological Society, Minnesota.

Lemonnier, P. (1992). Elements for an anthropology of technology, Ann Arbor, Michigan.

López, M. (2016). Integración social a nivel supra doméstico de las comunidades presentes en Isla Mocha durante el periodo Alfarero Tardio: Una aproximación a partir de los estilos tecnológicos de producción cerámica, Memoria para optar al título de Arqueóloga, Universidad de Chile, Santiago.

López, M.C., Gajardo, A., Carrasco, R. y J.L. Mendoza (2003). Yacimientos de rocas y minerales industriales de la VIII Región del Bío Bío. Servicio Nacional de Geología y Minería. Escala 1:500.000. Carta Geológica de Chile, Serie Recursos Minerales y Energéticos (16), 14.

Neff, H. (2012). Laser Ablation ICP-MS in archaeology, en: M.S. Lee (ed.), Mass spectrometry handbook, 829-843, John Wiley \& Sons, Hoboken.

Neff, H. (2017). LA-ICP-MS Analysis of pigments and slips from Southern Chile, Institute for Integrated Research in Materials, Environments, and Society, California State University, Long Beach.

Porter, B. y R. Speakman (2008). Reading moabite pigments with laser ablation ICP-MS: a new archaeometric technique for near eastern archaeology, Near Eastern Archeology 71(4), 238-242. https://doi.org/10.1086/ NEA20697195

Reyes, V. (2010). Microvariaciones en la cerámica de sitios alfareros tardíos de dos cuencas lacustres precordilleranas de la Araucanía: lagos Villarrica y Calafquén, IX y XIV Regiones de Chile, Memoria para optar al título de Arqueóloga, Universidad de Chile, Santiago. 
Roa, C., Silva, C. y R. Campbell (2012). El aporte de isla Mocha al conocimiento sobre el aprovechamiento de plantas con valor alimenticio en el sur de Chile (1000-1700 d.C.), Actas del XIX Congreso Nacional de Arqueología Chilena, 549-559.

Rosales, D. (1674[1877] Historia general del reino de Chile, Flandes Indiano, Tomo I, Imprenta del Mercurio, Valparaíso.

Sánchez, M. (1997). El período alfarero en la isla Mocha, en: D. Quiroz y M. Sánchez (eds.), La isla de las palabras rotas, 103-131, Dirección de Bibliotecas, Archivos y Museos, Santiago.

Sanhueza, L. (1997). Patrón cerámico: hacia la definición de un concepto operativo, Actas del XIV Congreso Nacional de Arqueología, 243-257.

Silva, C. (2014). Archaeobotanical remains, en: T. Dillehay (ed.), The teleoscopic polity. Andean Patriarchy and Materiality, 221-236, Springer, New York.

Speakman, R. y H. Neff (2002). Evaluation of painted pottery from te Mesa Verde región using Laser AblationInductively Coupled Plasma-Mass Spectrometry (LA-ICP-MS), American Antiquity 67(1), 137-144. https:// doi.org/10.2307/2694882

Stark, M. (1999). Social dimensions of technical choice in Kalinga ceramic traditions, en: E. Chilton (ed.), Material meanings. Critical approaches to the interpretation of material culture, 4-24, The University of Utah Press, Salt Lake City.

Weigand, P.C., Harbottle, G. y E.V. Sayre (1977). Turquoise sources and source analysis: Mesoamerica and the Southwestern USA, en: T. K. Earle y J. E. Ericson (eds.), Exchange systems in prehistory, 15-34, Academic Press, New York.

Fecha de recepción: 5-8-2019

Fecha de aceptación: 16-12-2019 